\title{
Portable Low-Power Electronic Interface For Explosive Detection Using Microcantilevers
}

\author{
D. García-Romeo ${ }^{a}$, I. Pellejero ${ }^{b}$, M.A. Urbiztondo ${ }^{\text {b,c }}$, J. Seséb ${ }^{\text {, M.P. Pina }}{ }^{\text {b,d }}$, P.A. Martínez ${ }^{a}$, B. \\ Calvo $^{a}$, N. Medrano ${ }^{a}$ \\ ${ }^{a}$ Group of Electronic Design, Aragon Institute for Engineering Research (I3A); University of Zaragoza; Facultad de \\ Ciencias, c/Pedro Cerbuna, 12, 50009 Zaragoza, Spain. \\ ${ }^{b}$ Nanoscience Institute of Aragón (INA), University of Zaragoza; Edif. I+D+i, Campus Rio Ebro, c/Mariano Esquillor \\ $\mathrm{s} / \mathrm{n}, 50018$ Zaragoza, Spain

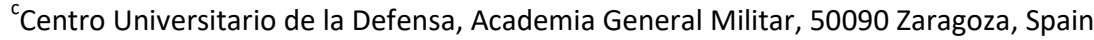 \\ ${ }^{d}$ Networking Research Center on Bioengineering, Biomaterials and Nanomedicine, CIBER-BBN, 50018Zaragoza, \\ Spain
}

\begin{abstract}
Microcantilevers have been recently revealed as highly effective sensing platforms for gas detection at trace level. However, one of the main limitations hindering their widespread deployment for commercial applications is related to the complexity of the read-out systems. A portable low-power electronic interface capable of creating the excitation signal as well as obtaining the response values of resonating Si microcantilevers functionalized with zeolite based coatings has been developed in this work. The accuracy and reliability of the electronic interface have been assessed by comparison with commercial lock-in amplifier's measurements. The prototype performance has been validated for 2-nitrotoluene (o-MNT) detection at ppm level, as an example of an explosive-related molecule. Theoretical limit of detection (LOD) values below 100 ppb have been obtained for Co exchanged BEA modified microcantilevers.
\end{abstract}

\section{Keywords}

Microcantilevers; gas sensing; zeolites; nanoporous coating; explosives; portable lock-in amplifier; low-power.

\section{Introduction}

Recent advances in chemical microcantilever-based sensors have led to a significant increase in sensitivity, making them a competitive solution in highly demanding applications as food and environmental security or explosives detection due to its ability to determine the presence and concentration of target gas species and volatile organic compounds (VOCs) [1-4]. Standard silicon microcantilevers working as tiny microbalances [5] achieve theoretical mass detection limits of around tens of $\mathrm{fg} / \mathrm{Hz}$. For dynamic mode operation, the analyte adsorption over the sensing layer deployed on top of the beam provokes an effective mass variation, which in turn modifies its natural oscillation frequency allowing sensitivities in the order of ppm [6-9]. Even so, its application on real multicomponent gas mixtures is challenging due to selectivity and cross-interference effects. These limitations are usually overcome by employing an array of sensors coated with different sensing materials. Thus, the presence and concentration of the 
target compounds can be determined through an appropriate pattern recognition algorithm with suitable accuracy and precision [10].

To accomplish miniaturized chemical microcantilever-based sensor arrays, the use of silicon provides important advantages in terms of shrinking power consumption and size. Our previous works [11-12] have shown that microcantilever type sensors coated with suitable zeolites leads to substantial outperformance in terms of sensitivity and selectivity.

Particularly, for early explosives detection, the main problem is related to their low vapor pressure in the pure form (i.e. between $10^{-2} \mathrm{~Pa}$ to $10^{-7} \mathrm{~Pa}$ at $25^{\circ} \mathrm{C}$ ). Thus, minor variations in the microcantilever's resonance frequency are expected, making difficult its subsequent readout. In this scenario, lock-in amplifiers (LIAs) are the most common solution on behalf of its versatile function recovering low and noisy signals [13-14]. Lock-in amplifiers are signal processing systems that use the phase sensitive detection (PSD) [15-16] technique to recover the data signal at a specific reference frequency; and reject noise signals at different frequencies without affecting the measurement significantly [17-18]. Nowadays, commercial lock-in amplifiers are easily found in any measurement laboratory. However, weight, cost and power consumption are the main drawbacks hindering their widespread use for portable sensing systems.

Thus, to exploit the truly benefits of the microcantilever-based sensor arrays in terms of portability, reduced size and energy consumption; an electronic readout interface showing similar features is required. In addition, the readout system must be flexible enough to be applied to the different elements constituting the sensor array. In fact, this is the main goal of the work. Firstly, the portable low-power electronic readout interface capable to measure Si microcantilever-based sensor output signals, with a precision up to $20 \mathrm{mHz}$ for the oscillation frequency measurement is presented. Thus, the microcantilever-like sensors used in this work are briefly described in Section 2. This section also highlights the electrical sensor requirements that the prototype must fulfill for a proper function. The design of the electronic interface powered through a universal serial bus (USB) is addressed in Section 3; where the analog lock-in amplifier, denoted as LIA for the remainder of the work, able to process square signals is fully explained. Such key element allows the read out of the low signals provided by the sensing platform. The experimental protocols either for determination or tracking the resonance frequency are described in Section 4 with special emphasis on the ProportionalIntegral-Derivative (PID) algorithm for high resolution measurements. The attained results are compared in Section 5 with those obtained with a commercial LIA model 7265 from Signal Recovery. Finally, the developed prototype is applied for 2-nitrotoluene detection, an explosive related molecule, with BEA zeolite coated Si microcantilevers. The evaluated performance demonstrates the potentialities of the sensing platform for detection at ppmV level.

\section{Microcantilever-based Gas Sensors}

Figure 1.a illustrates the typical layout for a microcantilever electromagnetically actuated. The microdevice is subjected to a constant magnetic field $\left(B_{\text {ext }}\right)$ originated by a permanent magnet placed below; then, an alternate current (I) passes through the microfabricated actuation wire, generating an alternate deflection force $\left(F_{L}\right.$ : Laplace force) that results into the mechanical 
oscillation. This oscillation is detected by a strain gauge located in the anchorage of the microcantilever.

The resonant oscillation frequency of a microcantilever tip varies according to its mechanical features. Theoretical frequency $f_{0}$ for a Si rectangular shape beam (Figure 1.a) can be calculated as follows:

$$
f_{o}=r_{n} \cdot \frac{t}{L^{2}} \cdot \sqrt{\frac{E}{\rho}}
$$

Where $t$ is the device thickness, $L$ is the length, $E$ is the Young's modulus (for $\mathrm{Si}<100\rangle, \mathrm{E}=130$ GPa), $\rho$ is the Si density $\left(2330 \mathrm{~kg} / \mathrm{m}^{3}\right)$ and $r_{n}$ depends on vibrational mode (for fundamental mode $\left.r_{n}=0,1615\right)$. Thus, the cantilever oscillation amplitude reaches a maximum when the frequency of the $A C$ current flowing through the actuation wire corresponds to the cantilever resonant frequency. Otherwise, the oscillation amplitude is progressively damped.

In this work, the cantilever oscillation movement is measured by a strain gauge composed by two identical piezoresistive elements connected in a half Wheatstone bridge, biased with a constant voltage. One of the piezoresistors is placed on the rigid substrate, while the other is located in the clamped-end of the beam, where stress is maximum. In this way, the oscillation movement of the cantilever acts over the corresponding piezoresistor changing its value. Thus, a variable voltage is generated at the bridge output, which frequency corresponds to the mechanical oscillation frequency of the cantilever. The resonance frequency is detected by measuring the maximum voltage amplitude and its corresponding phase out at the output of the strain gauge. Figure 1.b illustrates the electrical equivalent of this microcantilever sensor. It requires an excitation periodic signal through the actuation wire and two fixed voltages to feed the strain gauges in order to generate the sensor output $\mathrm{V}_{\text {sens }}$.

The Si microcantilever chip used in this work (Figure 1c) comprises 8-cantilevers, $200 \mu \mathrm{m}$ wide and $15 \mu \mathrm{m}$ thick, that have been coated with microporous zeolites. The deployment of suitable coatings on the mechanical transducers allows the adsorption of the target analytes. In particular, the commercial BEA type zeolites used in this work were provided by Zeolyst International ( $\mathrm{Si} / \mathrm{Al}$ ratio $=12,5 ; \mathrm{NH}_{4}{ }^{ \pm}$form). Cobalt ion exchange was performed following the recipe described in our previous works [9-12]. The Si cantilevers were microfabricated by D+T Microelectrónica A.I.E, according to our own design and specifications [11-12]. One of their main features is the incorporation of a heater wire on the cantilever tip for the fast temperature cycling required to sorbent regeneration. As the length of the beam varies from $500 \mu \mathrm{m}$ to $535 \mu \mathrm{m}$, the exhibited resonance frequency values range from 90 to $65 \mathrm{kHz}$. 

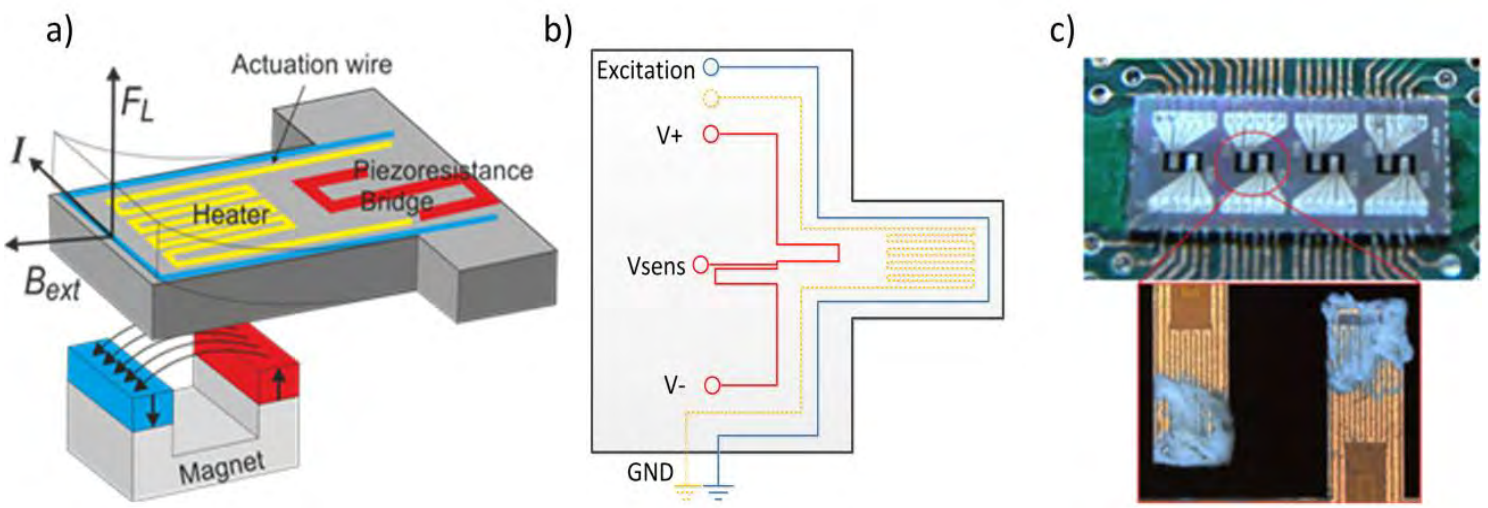

Figure 1.a) Schematic layout of dynamic operation mode for the cantilevers, with electromagnetic actuation, piezoresistive detection and integrated heater; b) Electrical diagram of the microcantilever sensor; c) Optical image of the 8 Si-microcantilevers array (200 $\mu \mathrm{m}$ wide, $15 \mu \mathrm{m}$ thick, $500 \mu \mathrm{m}$ to $535 \mu \mathrm{m}$ length) used in this work, the inset corresponds to the Si microcantilever tip after deposition of zeolite crystals by microdrop coating technique.

\section{Readout system}

Commercial LIAs are commonly used for both generating the exciting signals as to perform the sensor readout for lab scale characterization. In general, these instruments provide the suitable exciting signal to the actuation wire. In addition, they are capable to perform an accurate reading of small and noisy signals at the sensor output; allowing a precise selection of the operation frequency window. Unfortunately, the use of commercial LIAs as readout devices in real applications using cantilever-based gas sensor arrays becomes unpractical due to size and energy requirements.

In our particular case, a suitable readout system must generate in parallel eight different exciting signals at specific frequency values; which in fact, are continuously modified as a function of the gas phase composition and operating temperature. Furthermore, the sensor's electrical outputs should be measured. The eight sensors could be excited and measured using just one LIA by input and output multiplexing, respectively. However, the selection of the working cantilever among those comprising the array results in de-synchronization between measurements. One possible solution could rely on the use of eight LIAs in parallel; but size, cost and power consumption make it unfeasible. To address this issue, Figure 2 shows the block diagram of the proposed electronic interface prototype. It comprises three main blocks: i) a power supply that generates the DC levels required by both the sensor and the electronic interface; ii) a readout system based on a compact LIA; and, iii) a microcontroller that manages the system operation: DC excitation for the piezoresistors, AC current bias for the actuation wire, system temperature control, frequency selection for the lock-in signals, LIA output reading and host computer communications. Each of these main building units will be discussed next. 

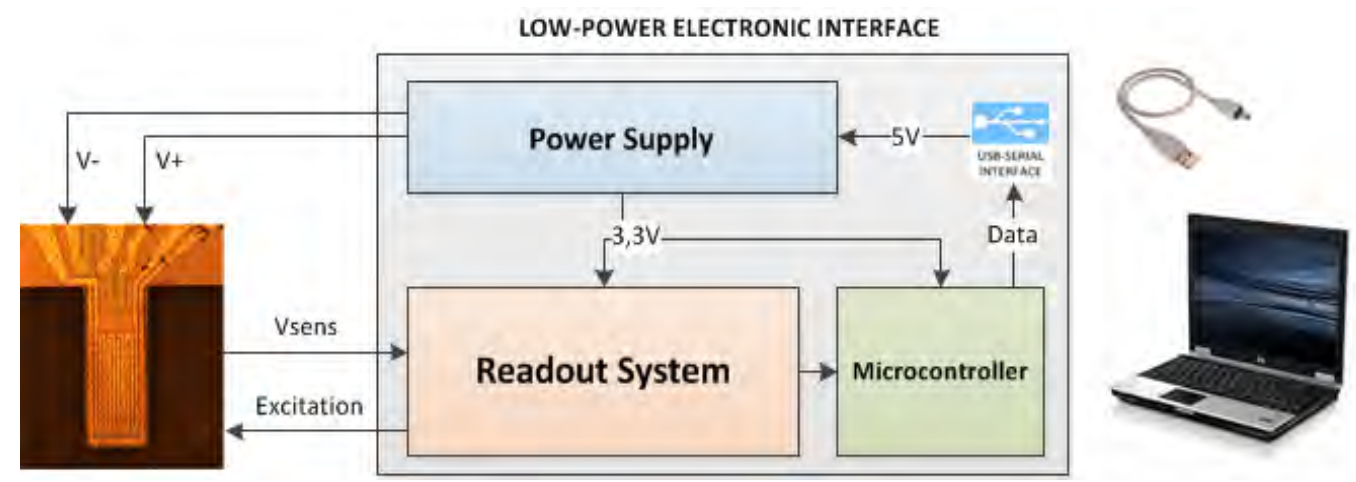

Figure 2. Block Diagram of the implemented low-power electronic interface.

\section{Power supply}

The $5 \mathrm{~V}$ line provided by the USB connector powers the prototype. Voltage levels are adapted using a voltage regulator that provides the 3,3 $\mathrm{V}$ suitable for the system electronics. Strain gauges use positive and negative DC bias voltage. Both levels are obtained using a LM2662 voltage doubler from Texas Instruments ( $\pm 9 \mathrm{~V}$ DC output) connected to an ASA00CC18-L DCDC Converter from Emerson Network Power to generate DC levels of $-15 \mathrm{~V}$ and $+15 \mathrm{~V}(\mathrm{~V}$ - and $V+$, Figure 2) to bias the Wheatstone bridge.

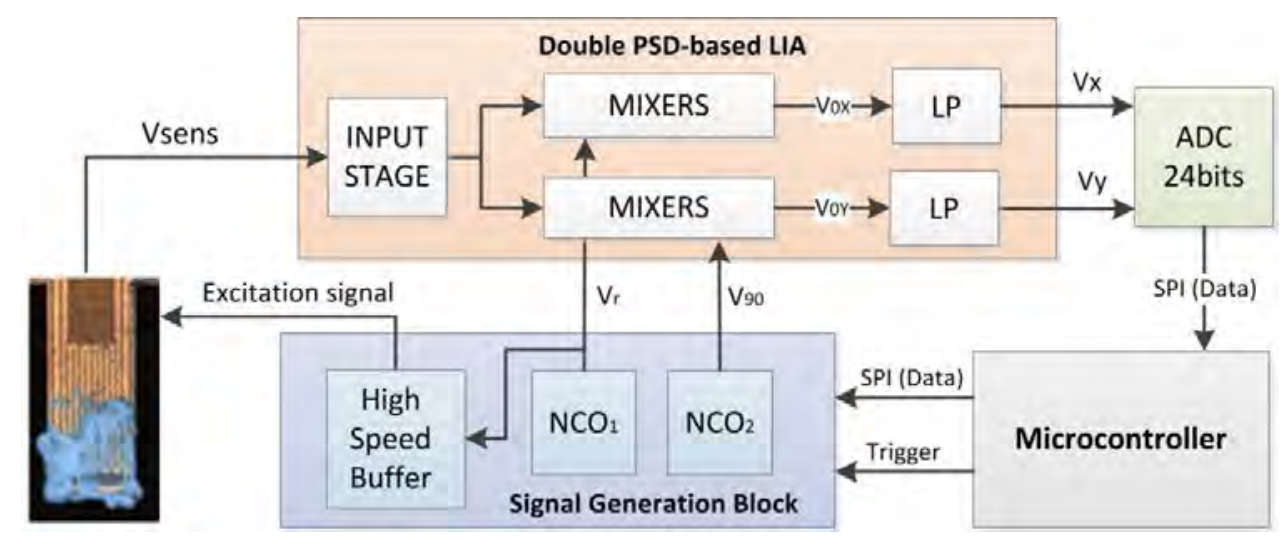

Figure 3. Block diagram of the readout block.

\section{Readout Block}

The core of the proposed portable readout block (Figure 3 ) is a low-voltage low-power dual PSD-based LIA [19-20] that carry out the measurement of the sensor output signal. An in-depth development of the dual PSD technique for processing of square signals, showing recovery equations for both amplitude and phase; and its experimental verification have been recently attempted by our group [21]. Besides, it comprises a signal generation block that generates the periodic signals at the operating frequency of the cantilever, and an ADC Stage for a precise digitization of the LIA outputs.

Some commercial integrated circuits, such as analog multipliers or demodulators [22], are amenable for assembly on a lock-in amplifier with appropriate additions. However, the complex configuration of the circuit, the supply voltages and consumption levels make them inappropriate for portable systems. Diverse interfaces suitable for sensor conditioning systems 
[23] can be found in the published literature. In general, these systems are designed on ad-hoc basis [24], that means specific operation frequency windows and voltage output. To the best of our knowledge, a general purpose portable lock-in amplifier has not been previously attempted. A preliminary version of the novel architecture based on the application of a dual PSD technique processing square signals instead of sinusoidal counterparts, was advanced by the authors earlier in [19-20]. Additional efforts have resulted in the herein used quasi-digital LIA capable of recovering of both amplitude and phase by means of a novel fully tested and validated algorithm [21]. The use of square signals for exciting sensor reduces electronics complexity, and accordingly, size and power consumption. Eventually, depending on the required frequency resolution, the system microcontroller can act as a square wave generator. All these features are clearly advantageous for the LIA implementation in portable systems that utilize single-supply battery cells.

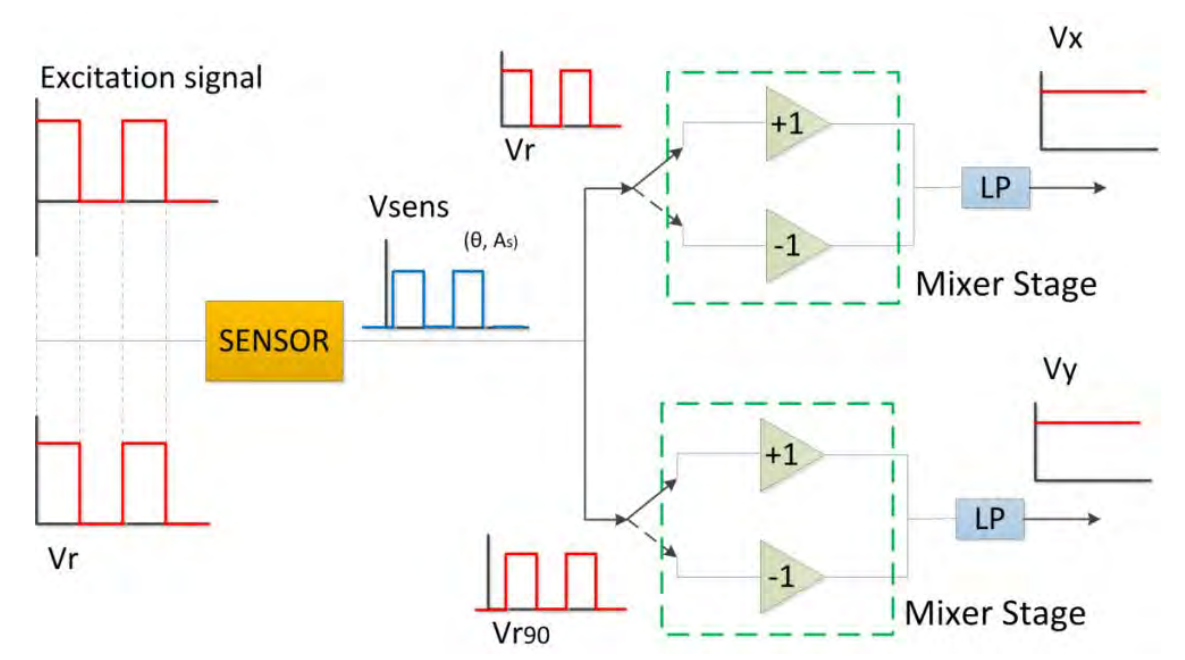

Figure 4. Block diagram of a two branches quasi-digital PSD system, each branch consisting of a mixer and low-pass filter.

In the designed LIA (Figure 4), the input signal provided by the sensor $V_{\text {sens }}$ enters to the Input Stage for pre-conditioning. This signal reaches both Mixer Stages, which are controlled by two reference square signals in quadrature $\left(V_{r}, V_{r 90}\right)$. Each mixer consists of two ADG719 2:1 multiplexers and a unity gain differential amplifier AD8476 from Analog Devices. Thus, $V_{o x}$ and $V_{o r}$ are the outputs from the mixers at each branch.The final stage corresponds to two low pass filters providing the DC signals $V_{X}$ and $V_{Y}$ that are required to obtain both magnitude $A_{S}$ and phase shift $\theta$. Low-pass filters are RC circuits with a cut-off frequency in the $\mathrm{Hz}$ range. These DC outputs $V_{X}$ and $V_{Y}$ are fed to identical 24-bit LTC2400 low-power ADC converters from Linear Technology. The designed algorithm uses $V_{X}$ and $V_{Y}$ to accurately calculate the output sensor signals parameters.

For microcantilever-based sensors operating in dynamic mode, an extremely accurate frequency determination becomes pivotal to ensure gas detection at low concentrations. Consequently, a specific signal generation block has been implemented to select the operation frequency window in a wide range with suitable accuracy and resolution. Signal generation is due to two AD9834 Numerically Controlled Oscillators (NCOs) from Analog Devices included in the prototype. NCOs are capable of generating square signals with frequencies from 1 to 100 
$\mathrm{kHz}$ with a resolution of $20 \mathrm{mHz}$ using a $10 \mathrm{MHz}$ quartz crystal as external reference. NCOs parameters are configured by the system microcontroller. Outputs from NCOs are two square wave signals in quadrature $\left(V_{r}, V_{r 90}\right.$, Figure 4$)$ that control the Mixer Stage of the implemented LIA. $V_{r}$ is also used as excitation signal of the sensor. Due to the low input impedance presented by the microcantilevers, a high speed unity-gain open-loop buffer BUF634 from Burr-Brown is used as output stage (see Figure 3).

\section{Microcontroller}

The electronic interface is controlled by a low-cost low-power Atmega1281 microcontroller from Atmel. It manages both ADCs and NCOs operation by SPI communication [26], selecting the frequency and phase values to the NCOs output signals and reading the data provided by the ADCs.After everymeasuring cycle the data are transferred from the microcontroller to the host computer throughan UB232R [25] Serial-to-USB adaptor from FTDI Chip.

Figure 5.a depicts the full system including the microcantilevers array connected to the herein presented interface. Figure 5.b shows a detailed image of the electronic interface.The consumption of the whole interface is $24 \mathrm{~mA}$ allowing the connection of more than 10 prototypes in parallel to the same USB port.

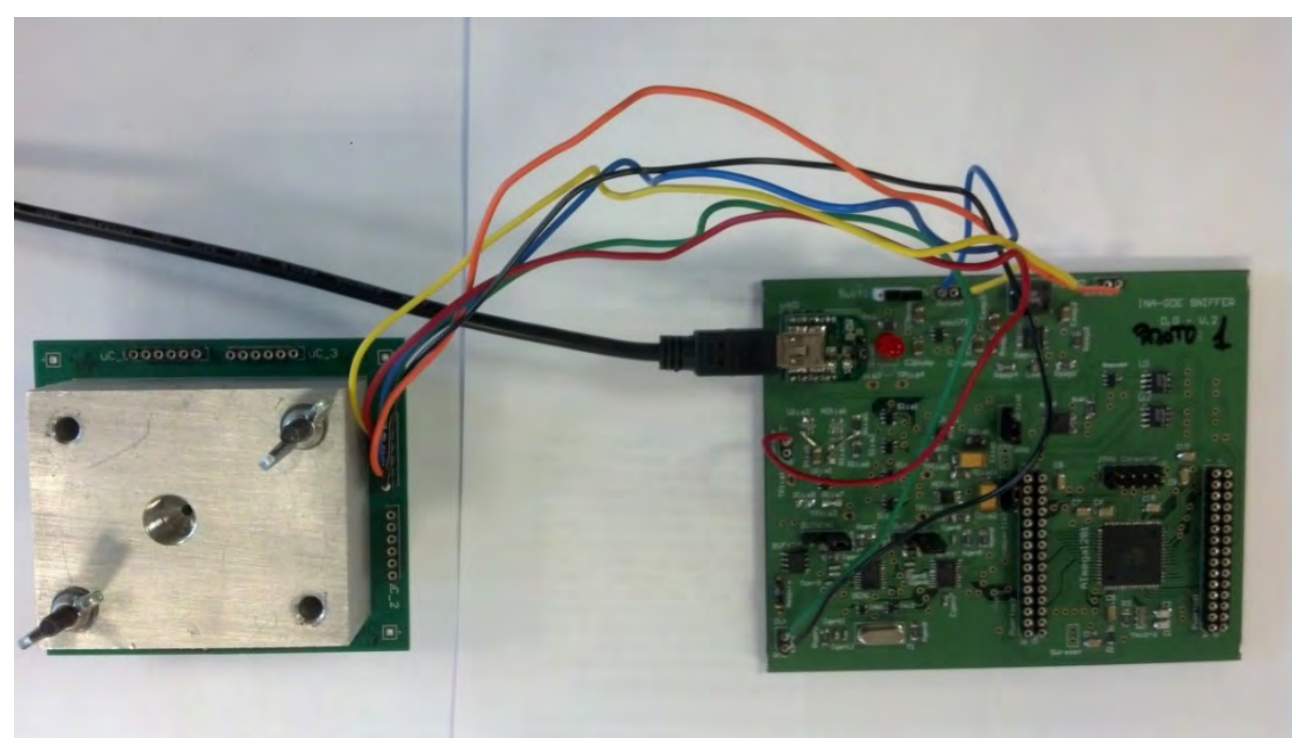

(a) 


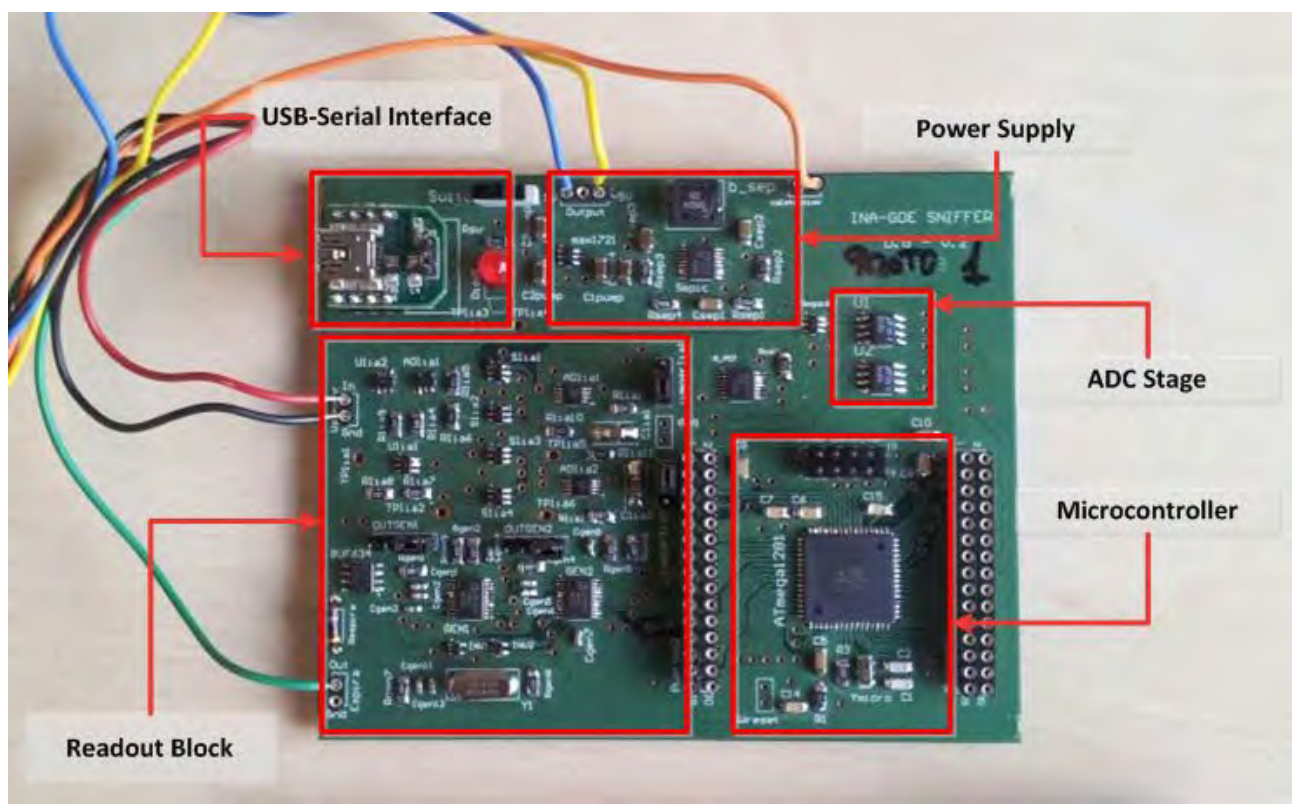

(b)

Figure 5. a) Portable Low-Power Electronic Interface for Si-microcantilever based sensors:

a) gas chamber for the sensing platform connected to the implemented interface; $b$ ) detailed image of the electronic interface $(110 \mathrm{~mm} \times 90 \mathrm{~mm})$.

\section{Measurement Protocoles}

\section{Natural frequency determination}

According to the behavior of a resonant oscillating system, the oscillation amplitude is maximal at the natural frequency $f_{o}$, where the characteristic phase change is also located. Consequently, the experimental $f_{o}$ value could be easily determined by carrying out a frequency sweep routine of both amplitude and phase parameters around the theoretical resonant frequency. Figure 6 shows a comparison of the amplitude (Figure 6.a) and phase (Figure 6.b) measurements of the microcantilever A5 (Table 1) performed with the proposed electronic interface and the commercial 7265 lock-in amplifier. Both systems have been configured to work at 1 step (data acquisition plus frequency actuation) per second. Note that in the proximity of the natural resonance, phase tracking is more sensitive than amplitude tracking for $f_{o}$ determination. It is also quite remarkable the similarity between both results. The natural frequency evaluated with the commercial LIA processing sinusoidal signals is $81531 \mathrm{~Hz}$ and the measured by our prototype working with square waves is $81529 \mathrm{~Hz}$. This deviation could be related to the differences in the sensor excitation signal: 1 Vrms vs. 3,3 V for commercial and portable LIA respectively. Furthermore, phase deviation out of the resonance region is due to the drastically reduced response of the sensor (Figure 6.a), that makes difficult a proper phase measurement. 


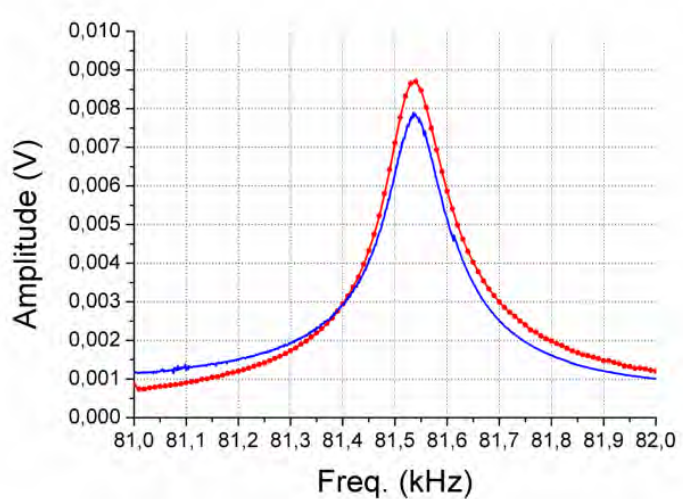

(a)

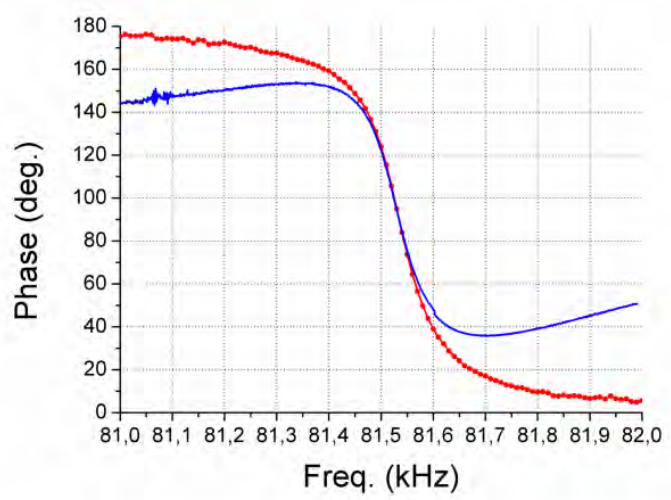

(b)

Figure 6. Amplitude (a) and phase (b) measurement with prototype (red dot) and commercial 7265 (blue line) LIAs near the natural resonance frequency for the microcantilever A5 (Table 1).

\section{Frequency tracking}

For gas sensing measurements, once the fundamental frequency has been determined in inert atmosphere, the system tracks the cantilever behavior and keeps its oscillation. To accomplish this, it modifies the excitation frequency in accordance to the effective mass variation of the beam due to the chemical interaction between the sensing material and the gas phase. Afterwards, these frequency shifts are correlated to the concentration of target analytes present in the atmosphere.

Frequency tracking for a given cantilever is performed by means of a classical PID algorithm. At the resonance, the sensitivity with respect to frequency changes is minimum for the amplitude and maximum for the phase (Figure 6). For that reason, we have implemented a PID algorithm to keep its phase constant during the whole gas sensing experiment by properly triggering the corresponding NCO.

The electronic interface is controlled by a graphical software (Figure 7) running on the host computer. For natural frequency determination in inert atmosphere (Figure7.a), the software requires three inputs from the user: initial and final frequency values and step size. Once the frequency sweep is accomplished, data are processed for the accurate determination of the natural frequency of the cantilever (amplitude and phase). In fact, such information (frequency value and target phase) has to be provided by the user to perform the tracking routine required for gas detection. By controlling the phase shift variations (Figure7.b bottom graph), the implemented algorithm modifies the sensor excitation signal to ensure cantilever oscillation at its natural resonance mode. As an example, Figure 7.b represents the typical resonance frequency evolution (top graph) with gas phase composition due to desorption/adsorption on target analytes on the cantilever tip. 


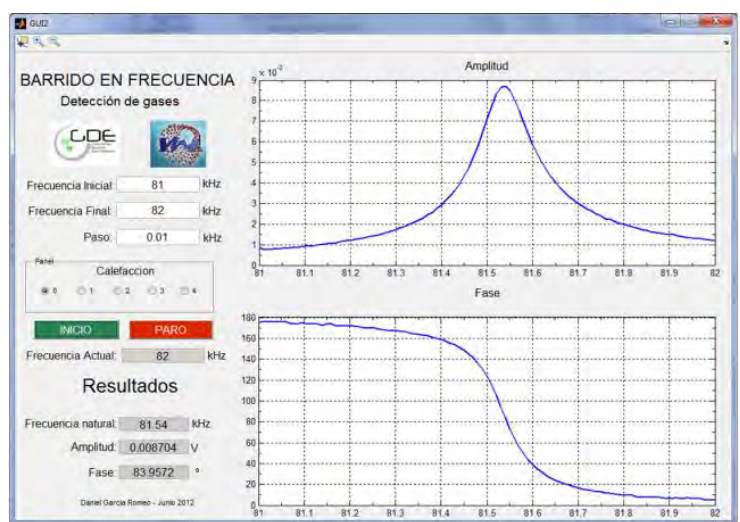

(a)

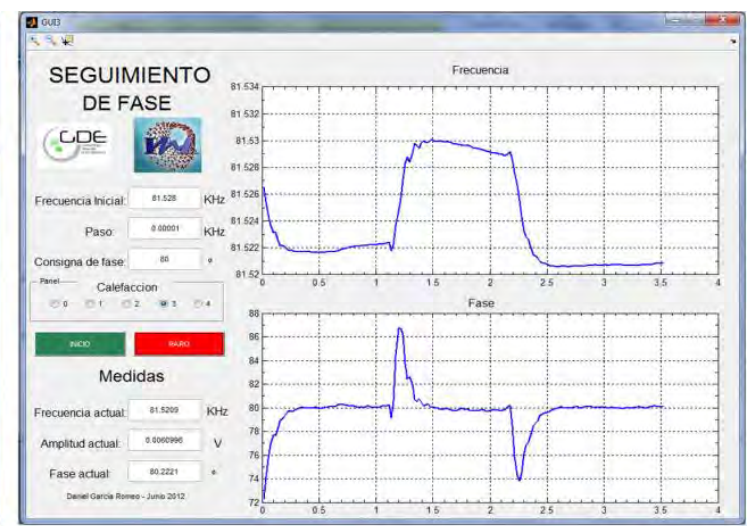

(b)

Figure 7. Implemented graphical user interfaces: (a) frequency sweep: amplitude (top graph) and phase (bottom graph) versus frequency, (b) frequency tracking: operating frequency (top graph) and phase (bottom graph) versus time on stream.

\section{Experimental results}

Several experiments have been carried out for prototype operation and validation. Firstly, resonance frequency values have been obtained for the 8-microcantilever chip shown in Figure 1. The results have been assessed by comparison with a commercial lock-in amplifier. The so obtained values prove the accuracy and reliability of the herein presented prototype. Secondly, the prototype performance for gas sensing has been demonstrated on zeolite functionalized cantilever exposed to different concentrations of 2-nitrotoluene (o-MNT), an explosive related molecule, in presence of humidity.

Table 1. Fundamental resonance frequency values for the 8 Si-microcantilevers estimated by commercial LIA 7265 and the prototype herein developed.

\begin{tabular}{|l|c|c|c|c|}
\cline { 2 - 5 } \multicolumn{1}{c|}{} & $\begin{array}{c}\text { Length } \\
(\boldsymbol{\mu m})\end{array}$ & $\begin{array}{c}\text { Theoretical } \\
\text { Frequency }\end{array}$ & $\begin{array}{c}\text { LIA7265 } \\
\text { Freq. (Hz) }\end{array}$ & $\begin{array}{c}\text { Prototype } \\
\text { Freq. (Hz) }\end{array}$ \\
\hline Cantilever A1 & 500 & 86868 & 84624 & 84621 \\
\hline Cantilever A2 & 535 & 75874 & 73415 & 73411 \\
\hline Cantilever A3 & 505 & 85156 & 83552 & 83555 \\
\hline Cantilever A4 & 530 & 77312 & 75325 & 75330 \\
\hline Cantilever A5 & 510 & 83495 & 81531 & 81529 \\
\hline Cantilever A6 & 525 & 78792 & 76748 & 76744 \\
\hline Cantilever A7 & 515 & 81881 & 80212 & 80217 \\
\hline Cantilever A8 & 520 & 80314 & 77532 & 77535 \\
\hline
\end{tabular}




\section{LIA Comparison}

The mechanical response of each sensor from the 8-microcantilever chip has been completely characterized by gathering both amplitude and phase at their fundamental frequencies with the custom LIA prototype. Table 1 comparatively shows these experimental frequencies values along with theoretically predicted ones and those acquired by a commercial LIA 7265 from Signal Recovery.

The theoretical frequency calculated with formula (1) is about $2 \%$ higher than the experimental value. Such deviation could be explained by a $2 \%$ variation of the Si beam thickness with respect to the nominal value. On the other hand, an excellent agreement between both LIA amplifiers is achieved.

\section{Vapor Explosive detection}

The herein described LIA prototype has been applied to gas sensing experiments using zeolite coated microcantilever. When microcantilevers are used as mass sensors, higher mass sensitivity $(\mathrm{S})(\mathrm{Hz} / \mathrm{ng})$ factors are pursued. Thus, little variations in the effective cantilever mass $(\Delta m)$, due to specific analyte adsorption or sensing material deposition, provokes noticeable frequency shifts $(\Delta f)$, (see equation 2 , where $f_{0}$ is the fundamental mode frequency; $m$ is the cantilever effective mass; $w$ denotes the width beam, i.e. $200 \mu \mathrm{m} ; L$ is the length; $E$ is the $S \mathrm{i}$ Young's modulus, i.e $130 \mathrm{GPa} ; \rho$ is the Si density, i.e $2330 \mathrm{~kg} / \mathrm{m}^{3}$; and $r_{n}=0,1615$ for fundamental mode $r_{n}$ ).

$$
S=\frac{\Delta f}{\Delta m}=-\frac{1}{2} \frac{f_{0}}{m}=-r_{n}^{-1} \cdot L^{3} w \sqrt{\frac{\rho^{3}}{E}}
$$

Accounting from our previous works [11-12] on zeolite coated cantilevers for explosives detection; some Si microcantilevers have been functionalized with BEA type zeolites by microdropping technique and tested with the LIA prototype. Cobalt (II) exchange procedure was applied on commercial BEA zeolite crystals in the ammonium form (Zeolyst CP814E) following the procedure described by [27] to modify its affinity towards 2-nitrotoluene vapors in presence of interferences. The exchanged powders exhibit a $\mathrm{Co} / \mathrm{Al}$ atomic ratio up to 0,76 (measure by EDX - energy dispersive X-ray spectroscopy), i.e. higher than the corresponding to $100 \%$ of exchanged $\mathrm{NH}_{4}{ }^{+},(\mathrm{Co} / \mathrm{Al}=0,5)$; indicating the existence of extra Co (II) species on the external surface of zeolite crystals. Table 2 summarizes the main characteristics of the selected modified Si-microcantilevers for the purposes of this work. Uncoated bare Si cantilever has also been included for comparison purposes.

The 8 Si-microcantilever chip was allocated in a Teflon ${ }^{\circledR}$ chamber with a total volume of $1 \mathrm{~mL}$, where gas composition measurements have been performed at $30^{\circ} \mathrm{C}$. Gas streams containing mixtures of 2-nitrotoluene vapors and nitrogen as a carrier gas were fed to the chamber at set flowrates by means of mass-flow controllers through explosive saturator trains at controlled temperature. The further dilution with a second mass-flow controlled stream of nitrogen allowed to reach the desired concentration levels (from $1 \mathrm{ppmV}$ to18 ppmV).

Before adsorption experiments, a zeolite regeneration step is carried out by using the heater integrated on tip. It enables the water or volatiles content removal from the zeolite 
micropores. In particular, average temperature values on the sensing coating above $150^{\circ} \mathrm{C}[11$ 12] are easily attained with the printed heater on tip. This procedure ensures the zeolite adsorption capabilities refreshment; and enlarges the life expectancy of our sensor device. Moreover, the heating of the cantilever during sensing could provide with a valuable tool to discriminate the presence of interferences, and consequently increase the final selectivity towards explosive related molecules.

Table 2. Main characteristics of the zeolite modified Si-microcantilever used in this work for 2nitrotoluene detection in vapor phase.

\begin{tabular}{|c|c|c|c|c|c|}
\cline { 2 - 6 } \multicolumn{1}{c|}{} & $\begin{array}{c}\text { Sensitivity } \\
\text { Hz/ng* }\end{array}$ & $\begin{array}{c}\text { Frequency }(\mathrm{Hz}) \\
\text { Before coating }\end{array}$ & $\begin{array}{c}\text { Sensing } \\
\text { Layer }\end{array}$ & $\begin{array}{c}\text { Frequency }(\mathrm{Hz}) \\
\text { After coating }\end{array}$ & $\begin{array}{c}\text { Sensing material } \\
\text { loading (ng) }\end{array}$ \\
\hline Cantilever B2 & 16.24 & 74020 & $\mathrm{NH}_{4} \mathrm{BEA}$ & 67450 & 404,5 \\
\hline Cantilever B3 & 19.31 & 84190 & CoBEA & 77140 & 365,0 \\
\hline Cantilever B8 & 17.69 & 78300 & None & None & None \\
\hline
\end{tabular}

*Calculated from equation 2.

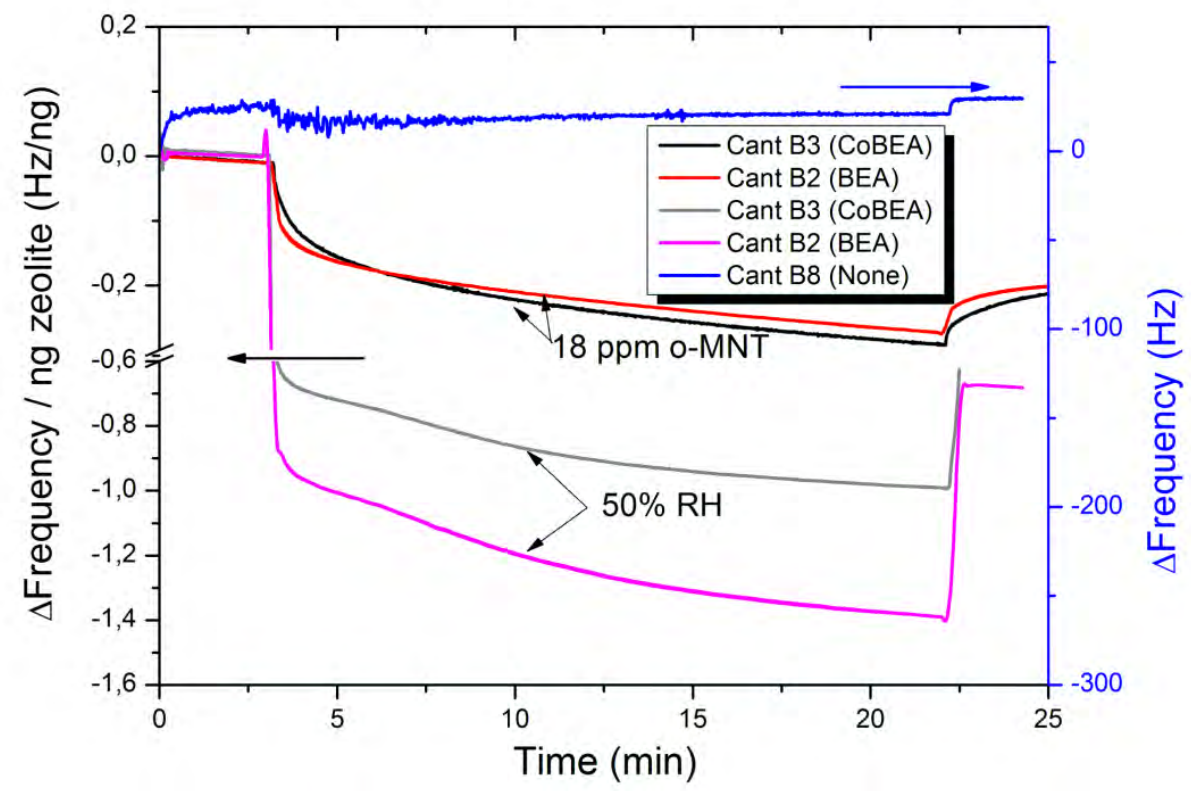

Figure 8. Evolution of the sensor response upon the introduction of 2-nitrotoluene (o-MNT) vapors and humidity at room temperature for cantilevers B3 (coated with Co-BEA) and B2 (coated with $\mathrm{NH}_{4} \mathrm{BEA}$ ). Response of bare Si cantilever $\mathrm{B} 8$ is also included as reference.

Figure 8 illustrates the frequency shift of $\mathrm{Si}$ microcantilevers modified with commercial $\mathrm{NH}_{4} \mathrm{BEA}$ (Cantilever B2) and CoBEA (Cantilever B3) type zeolites respectively in presence of humidity ( $50 \%$ of relative humidity ( $\mathrm{RH}$ ) at ambient conditions) and o-MNT as single analytes. As it can be observed, the sensor response has been normalized per sensing material loading 
for a proper data comparison. The performance of bare Si cantilever under identical conditions has also been included as reference. In spite of the huge differences in the gas phase concentration for the tested analytes (20000 ppmV $\mathrm{H}_{2} \mathrm{O}$ vs $18 \mathrm{ppmV}$ o-MNT), the zeolite modified sensor responses upon the introduction of explosive related molecule are only two fold lower than the obtained after water exposure. This behavior highlights the specific nitroaromatic sorption over the selected zeolites. It is also noteworthy the mechanical response of bare $\mathrm{Si}$ cantilever, almost unaffected by the gas composition. Received $\mathrm{NH}_{4} \mathrm{BEA}$ zeolites exhibit higher water adsorption capability than Co exchanged counterparts. This differential behavior is attributed to extra-framework $\mathrm{Co}^{2+}$ cations and also extra cobalt species on the external zeolite surface. This presence modifies the water adsorption properties; and, eventually, hinders the diffusion of water molecules to the micropores by steric effect. On the contrary, 2-nitroderivate sorption is rather similar for both BEA type zeolites. Thus, it can be concluded thato-MNT/water selectivity has been notably improved by the Co exchange process.

Figure 9 shows the resonant frequency evolution for the COBEA modified microcantilever upon the introduction of 2-nitrotoluene (o-MNT) steps $3 \mathrm{~min}$ in duration varying from $12 \mathrm{ppmV}$ to 1 ppmV. These experiments were performed to evaluate the kinetics and sorption capability on the basis of specific target-zeolite interactions. Once the feed is switched to inert $\mathrm{N}_{2}$ gas, the frequency response of the resonating microcantilever does not recover the initial value due to strong interactions between BEA type and 2-nitrotoluene vapors whatever the concentration tested. This behavior is clearly indicating the importance of an adequate sensor conditioning step when zeolites are used as sensing material.

From this set of vapor related explosive experiments, the as calculated o-MNT gas sensitivity (expressed in $\mathrm{Hz} / \mathrm{ppmV}$ ) by proper fitting of the experimental points (not shown here) is $\mathbf{4 0}$ $\mathrm{Hz} / \mathrm{ppm}$. In this work, the lowest concentration of o-MNT experimentally measured has been 1 ppmV due to practical limitations in the vapor generation system. However, accounting from the experimental signal noise (about $1 \mathrm{~Hz}$ ), theoretical limit of detection (LOD) values below $100 \mathrm{ppb}$ are obtained for B3 cantilever.

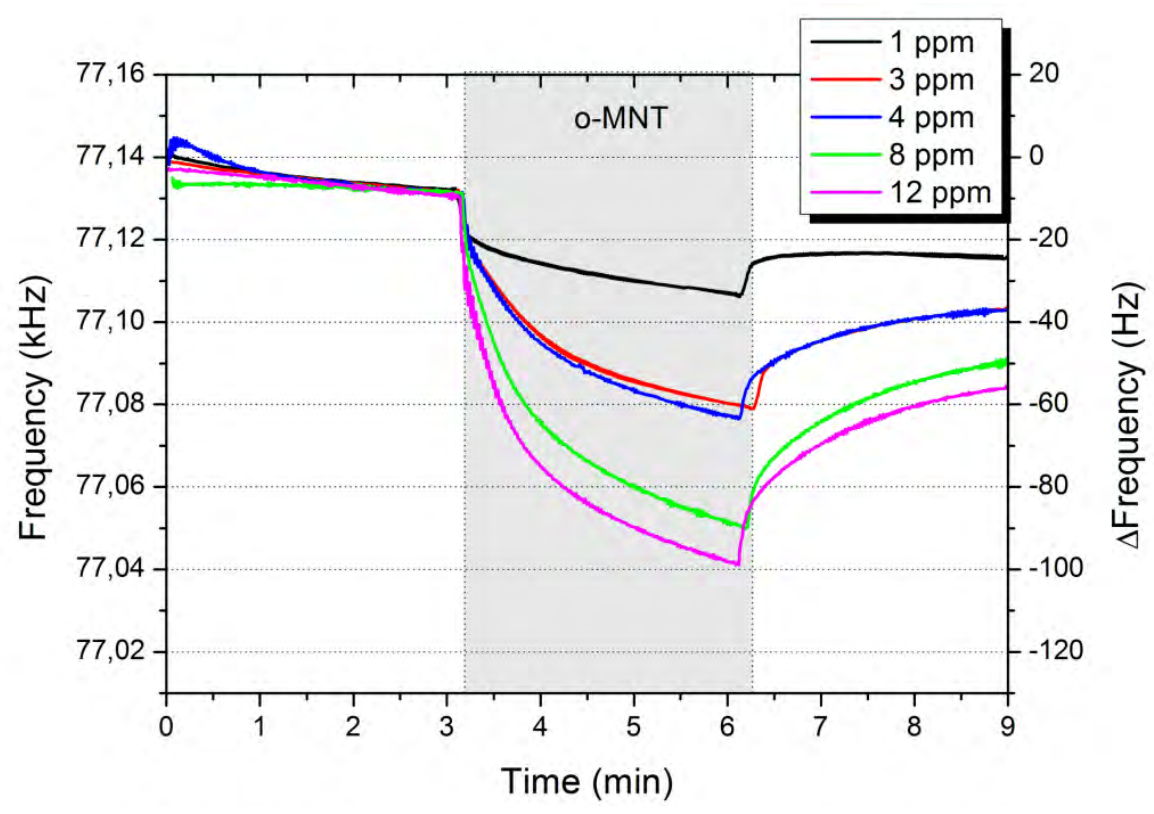


Figure 9. Evolution of the resonant frequency upon the introduction of 2-nitrotoluene (o-MNT) vapors at room temperature for $B 3$ cantilever.

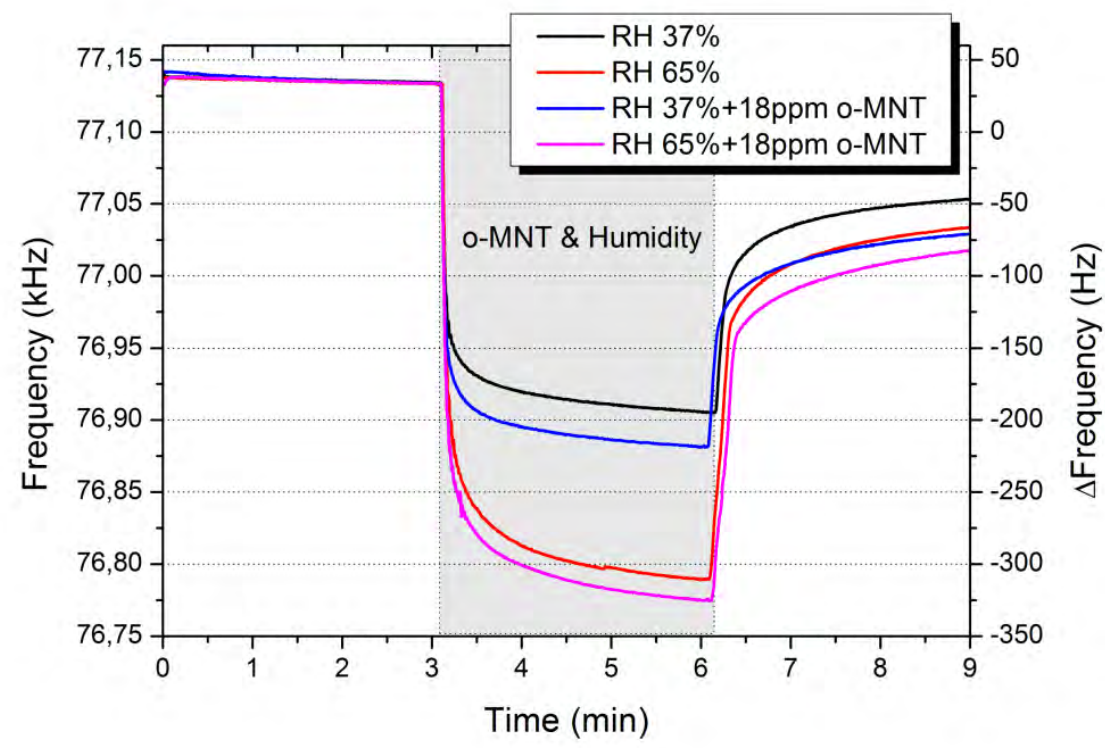

Figure 10. Evolution of the resonant frequency upon the introduction of binary mixtures of 18 ppmV of o-MNT vapors at two different relative humidity $(R H)$ values as interference for $B 3$ cantilever.

The characterization of the sensors herein prepared in presence of humidity as interference is crucial due to the hydrophylic properties of zeolites. Therefore, additional experiments with mixtures containing o-MNT (18 ppmV) and $\mathrm{H}_{2} \mathrm{O}$ vapors (15000 ppmV circa 7\% $\mathrm{RH}$, or 26000 ppmV circa 65\% RH) have been accomplished on B3 cantilever. The obtained results are depicted in Figure 10 where single $\mathrm{H}_{2} \mathrm{O}$ vapors ( $37 \% \mathrm{RH}$ and $65 \% \mathrm{RH}$ ) detection experiments are also included for comparison. The most outstanding feature is the frequency shift, higher than $100 \mathrm{~Hz}$; and attributed to the presence of $18 \mathrm{ppmV}$ of o-MNT even at $65 \% \mathrm{RH}$. This observation is again underlying the specific adsorption properties of the zeolite based sensing materials towards the target analytes in presence of interferences.

\section{Conclusions}

A portable low-power electronic interface for a multi-sensing platform comprising 8microcantilever has been successfully demonstrated in this work. The core element is a square signal based analog lock-in amplifier based on a dual phase technique that uses two reference signals in quadrature which enables the acquisition of both amplitude and phase measurements regardless of phase deviations produced by the sensors. This configuration together with a new algorithm capable of handling square signals constitutes a clear innovation for portable sensing systems. Specific software implementation has been developed to accomplish frequency sweeps at the desired frequency range and to enable dynamic mode operation with the microcantilever oscillating at its natural frequency. The accuracy of the LIA prototype has been proved by comparison with a commercial 7265 lock-in 
amplifier from Signal Recovery at reference conditions. The portable electronic interface, solely fed by the $5 \mathrm{~V}$ line from a USB connection to a host computer, has been validated for 2nitrotoluene detection, an explosive related molecule, by using BEA type zeolite modified microcantilevers. In particular, LOD values below 100 ppb for CoBEA modified cantilevers have been obtained.

The LIA prototype described in this work presents clear advantages in terms of versatility, computational simplicity, reduced size and energy consumption. Overall, the simplification of the cumbersome instrumentation paves the way for field experiments under relevant conditions. All these features also facilitate an easy implementation of our customized LIA to any other portable multi-sensing platform whatever the transduction schemes involved.

\section{Acknowledgements}

Financial support from MINECO through CTQ 2010-19276, TEC2012-30802 and RYC-200803185is gratefully acknowledged.

\section{References}

[1] A. Boisen, S. Dohn, S.S. Keller, S. Schmid, M. Tenje, Cantilever-like micromechanical sensors, Reports on Progress in Physics 74 (2011) 1-31.

[2] B. Rogers, R. Whitten, J.D Adams, Multifunctional self-sensing microcantilever arrays for unattended detection of chemicals, explosives, and biological agents. Unmanned/Unattended Sensors and Sensor Networks III. Edited by Carapezza, Edward Proceedings of the SPIE, 6394(2006).

[3] L. Senesac, T. Thundat, Nanosensors for trace explosive detection, MaterialsToday, 11(2008) 28-36.

[4] L. Senesac, D. Yi, A. Greve, J.H. Hales, Z.J. Davis, D.M. Nicholson, A. Boisen, T.Thundat, Micro-differential thermal analysis detection of adsorbed explosive molecules using microfabricated bridges, Review of Scientific Instruments, 80(2009) 1-9.

[5] F.M. Battiston, J.-P. Ramseyer, H.P. Lang, M.K. Baller, Ch. Gerber, J.K. Gimzewski, E. Meyer, H.-J. Guntherodt, A chemical sensor based on a microfabricated cantilever array with simultaneous resonance-frequency and bending readout, Sensors and Actuators B: Chemical, 77(2001) 122-31.

[6] D. Lange, C. Hagleitner, A. Hierlemann, O. Brand, H. Baltes, Complementary metal oxide semiconductor cantilever arrays on a single chip: mass sensitive detection of volatile organic compounds, Analytical Chemistry, 74(2002) 3084-95.

[7] C. Vancura, M. Ruegg, Y. Li, C. Hagleitner, A. Hierlemann, Magnetically actuated complementary metal oxide semiconductor resonant cantilever gas sensor systems, Analytical Chemistry, 77(2005) 2690-2699.

[8] M. Urbitzondo, M.P. Pina, J. Santamaría, in Ordered Porous Solids: Gas Sensing with Silicon-Based Nanoporous Solids, S Valtchev, S Mintova, M Tsapatsis (Eds), Elsevier, (2008) 381-405. 
[9] M.A. Urbiztondo, I. Pellejero, M. Villarroya, J. Sese, M.P. Pina, I. Dufour, J. Santamaria. Zeolite-modified cantilevers for the sensing of nitrotoluenevapors, Sensors and Actuators B: Chemical, 137 (2009) 608-616.

[10] F. Rock, N. Barsan, U. Weimar. Electronic Nose: current status and future trends, Chemical Reviews, 108(2008) 705-725.

[11] M.P. Pina, I. Pellejero, M.A. Urbiztondo, J. Sesé, J. Santamaría, Explosives detection using nanoporous coatings, Proceedings SPIE 8031(2011).

[12] M.A. Urbiztondo, A. Peralta, I. Pellejero, J. Sese, M.P. Pina, I. Dufour, J. Santamaria. Detection of organic vapours with Si cantilevers coated with inorganic (zeolites) for organic (polymer) layers, Sensors and Actuators B: Chemical 171- 172 (2012) 822-831.

[13] Sklorz, Adam; Janssen, Steffen; Lang, Walter,Detection limit improvement for NDIR ethylene gas detectors using passive approaches, Sensors and Actuators B: Chemical, 175(2012) 246-254.

[14] Melih Okan, Osman Balci, Coskun Kocabas,A microfluidic based differential plasmon resonance sensor, Sensors and Actuators B: Chemical, 160(2011) 670-676.

[15] D. P. Blair, Phase sensitive detection as a means to recover signals buried in noise, Phys E: Sci Instrum, 8(1975) 621-627.

[16] J.H. Scofield, Frequency-domain description of a lock-in amplifier, Am J Phys, 62(1994) 129-133.

[17] M.L. Meade, Lock-in Amplifiers: Principles and Applications, Peter Peregrinus Ltd, (1983).

[18] Lock-in amplifiers, application notes., Stanford Res, datasheets(1999).

[19] J. Aguirre, N. Medrano, B. Calvo, S. Celma, Lock-in amplifier for portable sensing systems, Electronics Letters, 47(2011).

[20] J. Aguirre, N. Medrano, B. Calvo, S. Celma, A $3 \mathrm{~V}$ single supply LIA for portable sensing systems, Proceedings of IEEE Sensors, (2011) 1866-1869.

[21] J. Aguirre,D. Garcia-Romeo, N. Medrano, B. Calvo, S. Celma, Square Signal Based Algorithm for Analog Lock-in Amplifiers, IEEE Transactions on Industrial Electronics, DOI 10.1109/TIE.2014.2300054 (2014).

[22] Analog Devices Inc., AD630 datasheet.

[23] F. Reverter, O. Casas, A microcontroller-based interface circuit for lossy capacitive sensors, Measurement Science and Technology, 21(2010).

[24] A. D’Amico, A. De Marcellis, C. Di Carlo, C. Di Natale, G. Ferri, E. Martinelli, R. Paolesse, V. Stornelli, Low-voltage low-power integrated analog lock-in amplifier for gas sensor applications, Sensors and Actuators B: Chemical, 144(2010) 400-406.

[25] UB232R USB Mini-B FT232R, Evaluation Module Datasheet FTDI.

[26] Freescale Semiconductor, SPI Block Guide V03.06 (2000).

[27] A. Mihaylova, K. Hadjiivanov, S. Dzwigaj, M. Che, Remarkable effect of the preparation technique on the state of cobalt ions in BEA zeolites evidenced by FTIR spectroscopy of adsorved CO and NO, TPR and XRD, The Journal of Physical Chemistry B, 110(2006) 19530-19536.

\section{Biographies}

Daniel Garcia-Romeo graduated in ElectronicsEngineering from the Universityof Zaragoza. He is currently a Ph.D. student at the Group of Electronic Design at the Aragon Institute for Engineering Research (GDE-I3A) of the University of Zaragoza.His research interests include integrated sensor interfaces, signal processingand intelligent instrumentation. 
Ismael Pellejero received his Ph.D. (2012) degree in chemical engineering from the University of Zaragoza. He is currently a Research Associate in the Departmentof Chemical and Environmental Engineering and also works in the Nanoscience Institute of Aragon (INA). His research interests are nanostructured material and microsystems fabrication, mainly sensors and micromembranes.

Miguel Urbiztondo received his Ph.D. (2008) degree in Chemical Engineering from the University of Zaragoza. He is currently a Research Associate in the Department of Chemical and Environmental Engineering and also works in the Nanoscience Institute of Aragón (INA). His research interests are in the area of Nanoporous materials, particularly in microfabricatedzeolitic devices for chemical sensor applications.

Javier Sesé obtained his Physics Ph.D. in 1999 in the University of Zaragoza on the field of applied superconductivity in QuantumMetrology. During 2000-2004 he was working in the development of new MEMS devices for electrical metrology in the MESA+ Institute for Nanotechnology, The Netherlands. Back in Spain, in 2004 he was awarded a Ramón y Cajal contract in the Nanoscience Institute of Aragón (INA) where he is in charge of the laboratory of nanolithography. Currently his research interest includes the development of MEMS for gas sensors, biosensors, and applied superconductivity in very low temperature experiments. Since 2009, he is Lecturer at the Department of Condensed Matter Physics in the University of Zaragoza

Maria Pilar Pina Iritia is a Junior Lecturer (2007) in the Department of Chemical and Environmental Engineering at the University of Zaragoza. Her current research lines pivoted on microsystems development based on nanostructured materials for reaction, separation and sensing applications. She has participated in more than 38 research projects, in 15 of them as principal researcher, at national and international level. She is coauthor of 3 patents, 6 book chapters, 40 indexed scientific papers and more than 120 communications to scientific congresses. She has supervised 5 doctoral theses.

Pedro A. Martínezwas born in Zaragoza, Spain. He received the B. Sc. degree in 1971 and the Ph.D. degree in 1974 both in Physics from the University of Zaragoza, Spain. Since 1971 he has been with Department of Electronic Engineering and Communications at the University of Zaragoza where he is Professor. His research interest lies in the area of solid-state circuits, including analog IC design, non-linear networks, modeling of analog integrated circuits and current-mode signal processing.

BelénCalvoreceived the B. Sc. degree in Physics in 1999 and the Ph. D. degree in Electronic Engineering in 2004, both from the University of Zaragoza, Spain. She is Senior Researcher at the Group of Electronic Design at the Aragon Institute for Engineering Research (GDE-I3A) of the University of Zaragoza. Her research interests include low-voltage low-power analog and mixed-mode CMOS design strategies, implementation and test of reconfigurable smart sensorto-microcontroller ASIC interfaces, wireless sensors networks and wideband front-end IC transceivers.

Nicolás Medrano received the B.Sc. and Ph.D. degrees in physics from the University of Zaragoza, Zaragoza, Spain, in 1989 and 1998, respectively. He is currently an Associate Professor of Electronics with the Faculty of Physics and a Member of the Group of Electronic Design, Aragon Institute ofEngineering Research, University of Zaragoza. His current research interests include adaptive signal processing, integrated sensor interfaces, wireless sensor networks, and intelligent instrumentation. 